\title{
Macrophagic enhancement in optical coherence tomography imaging by means of superparamagnetic iron oxide nanoparticles
}

\author{
Juan Luis Gutiérrez-Chico ${ }^{1,2}$, Milosz Jaguszewski², Miguel Comesaña-Hermo ${ }^{3}$, \\ Miguel Ángel Correa-Duarte ${ }^{3}$, Luis Mariñas-Pardo ${ }^{4}$, Manuel Hermida-Prieto ${ }^{5}$ \\ ${ }^{1}$ Department of Interventional Cardiology, DRK-Klinikum Westend, Berlin, Germany \\ ${ }^{2}$ Institute of Cardiovascular Translation Research of the Atlantic (ICTRA), Berlin, Germany \\ ${ }^{3}$ Department of Physical Chemistry, Singular Centre for Biomedical Research (CINBIO), \\ Southern Galicia Institute of Health Research (IISGS), Vigo Universidade de Vigo, Spain \\ ${ }^{4} \mathrm{R} \& D$ Deptartment, Centauri Biotech, La Coruña, Spain \\ ${ }^{5}$ Instituto de Investigación Biomédica de A Coruña (INIBIC), Complexo Hospitalario \\ Universitario de A Coruña (CHUAC), Universidade da Coruña (UDC), Spain
}

This paper was guest edited by Prof. Lillian Grigorian-Shamagian

\begin{abstract}
Background: The ability of optical coherence tomography (OCT) to visualise macrophages in vivo in coronary arteries is still controversial. We hypothesise that imaging of macrophages in OCT could be enhanced by means of superparamagnetic nanoparticles.

Methods: We compared the optical backscattering and attenuation of cell pellets containing RAW 264.7 macrophages with those of macrophagic cell pellets labelled with very small superparamagnetic oxydised nanoparticles (VSOP) by means of light intensity analysis in OCT. The labelled macrophages were incubated with VSOP at a concentration of $1 \mathrm{mM} \mathrm{Fe}$, corresponding to intracellular iron concentrations of $8.8 \mathrm{pg} / \mathrm{cell}$. To study the effect of intracellular accumulation on the backscattering, VSOP dilutions without cells were also compared. OCT pullbacks of the PCR tubes containing the cell pellets were obtained and light intensity analysis was performed on raw OCT images in polar view, after normalisation by the backscattering of the PCR tube. The backscattering was estimated by the peak normalised intensity, whilst the attenuation was estimated by the number of pixels between the peak and the normalised intensity 1 (peak-to-one).

Results: VSOP-loaded macrophages have higher backscattering than the corresponding unlabelled macrophages (peak normalised intensity 6.30 vs. 3.15) with also slightly higher attenuation (peak-toone 61 vs. 66 pixels). The backscattering of the nanoparticles in suspension was negligible in the light intensity analysis.

Conclusions: VSOP increase significantly the optical backscattering of macrophages in the nearinfrared region, with minimal increase in signal attenuation. This finding enables the enhancement of macrophages in conventional OCT imaging with an easily implementable methodology. (Cardiol J 2017; 24, 5: 459-466)
\end{abstract}

Key words: plaque, atherosclerotic, coronary artery disease/physiopathology, inflammation, macrophages, ferrosoferric, oxide/diagnostic use, very small iron oxide particles, tomography, optical coherence

Address for correspondence: Prof. Juan Luis Gutiérrez-Chico, Interventional Cardiology, DRK-Klinikum Westend, Spandauer Damm 130, 14050 - Berlin, Germany, tel: +49 (0) 176 30585019, fax: +34 615319370 , e-mail: juanluis.gutierrezchico@ictra.es

Received: 04.05.2017 Accepted: 08.05.2017 


\section{Introduction}

Atherosclerosis is still the first cause of mortality in the world, especially in developed countries [1-3]. This mortality is mainly driven by the most severe clinical manifestations of atherosclerosis, namely stroke and myocardial infarction, that have a common pathological substrate in most cases: plaque destabilisation $[4,5]$. Inflammation and particularly macrophages play a relevant role in both atherogenesis and plaque destabilisation [6-11]. Macrophages and foam cells are directly involved in the progression of the plaque and its necrotic core $[8,11]$. Furthermore, macrophages can release matrix metalloproteinases with proteolytic activity, which digest the extracellular matrix and weaken the fibrous components of the cap, thus predisposing it to rupture [7,10,12-15]. Nonetheless the clinical evidence about the involvement of macrophages in plaque progression and plaque stability is still indirect at a large extent: most studies are based in animal models [14], necropsies $[6,12,13]$ or rare human specimens harvested during directional atherectomy (an interventional technique not performed in coronary vessels any more) $[7,10]$ or heart transplantation [15]. There are scant direct in vivo morphologic studies of macrophages in coronary atherosclerosis in a clinical setting that enable the sequential exploration of a process dynamic in nature.

Optical coherence tomography (OCT) has revolutionised intravascular imaging due to its high resolution and its high signal-to-noise ratio, that enables very fast and very accurate acquisition of a sizeable amount of information [16]. The use of in vivo OCT in clinical settings has provided unique insights into the pathogenesis of coronary atherosclerotic plaques [16], including the detection of inflammatory cells like macrophages [17]. The size of macrophages (around $20-50 \mu \mathrm{m}$ in diameter) is above the axial resolution of OCT $(14 \mu \mathrm{m})$ and their large nuclei backscatter the near-infrared (NIR) radiation significantly, therefore intimal accumulation of macrophages can result in a typical OCT image pattern. Nonetheless the sensitivity and specificity of unaided conventional OCT for the detection of macrophages are still quite controversial, so OCT has been granted only with a medium level of evidence for this specific aim in the last consensus about OCT image interpretation [18].

Several superparamagnetic iron oxide nanoparticles used as contrast agents for magnetic resonance imaging (MRI) have shown accumulation in macrophages of atherosclerotic plaques
[19-23], among them very small iron oxide particles (VSOP) that accumulate as agglomerates in phagolysosomes [21]. VSOP are rich in iron, which increases significantly the backscattering and attenuation of NIR radiation. Moreover, according to Rayleigh's theory, when the particle size is substantially lower than the light wavelength, the light scattering intensity is proportional to the $6^{\text {th }}$ power of the particle size and aggregation can be monitored by light scattering intensity [24]. Since the NIR radiation used for OCT has a wavelength between 700 and $1000 \mathrm{~nm}$ and the VSOP are below $20 \mathrm{~nm}$ diameter, we hypothesise that the accumulation and formation of aggregates of VSOP in macrophages might increase the backscattering and attenuation of NIR radiation and therefore enhance their visualisation in OCT imaging.

\section{Methods}

We compared the optical backscattering and attenuation of cultured control macrophages with those of macrophages loaded with citrate-coated very small superparamagnetic iron oxide particles (VSOP). An additional sample with VSOP in suspension, without cellular culture, was used also as control.

Very small superparamagnetic iron oxide particles are electrostatically-stabilised citrate-coated very-small iron oxide particles. Although they are being currently clinically tested as contrast agent for visualisation of atherosclerotic plaques in MRI $[22,23]$, they are not approved yet for clinical use. Nonetheless VSOP might have interesting pharmacokinetic advantages, because they cumulate directly in phagolysosomal structures of endothelial cells and macrophages, thus enabling imaging of inflammation in atherosclerotic plaques within $3 \mathrm{~h}$ after intravenous administration [20,25]. VSOP were synthesised following the well-known coprecipitation method [26]. Briefly, $7 \mathrm{~g}$ of $\mathrm{FeCl}_{2}$ and $12.35 \mathrm{~g}$ of $\mathrm{FeCl}_{3} 6 \mathrm{H}_{2} \mathrm{O}$ were separately solubilised in $50 \mathrm{~mL}$ of distilled water and then mixed together at $2^{\circ} \mathrm{C}$. Subsequently, $45 \mathrm{~mL}$ of $28 \% \mathrm{NH}_{3}$ were poured into the reaction flask and the solution immediately turned black due to the formation of VSOP. After this, the reaction was kept $1 \mathrm{~h}$ more at $2^{\circ} \mathrm{C}$ under mechanical agitation. Once the synthesis of VSOP was completed, their surface was modified with sodium citrate molecules. In order to do this, $150 \mathrm{~mL}$ of a $0.1 \mathrm{M}$ sodium citrate trihydrate solution were added to the as-synthesised nanoparticles and the solution was agitated for $1 \mathrm{~h}$ at $80^{\circ} \mathrm{C}$. After this, the product was isolated with the help of a permanent magnet and washed with distilled water. This puri- 


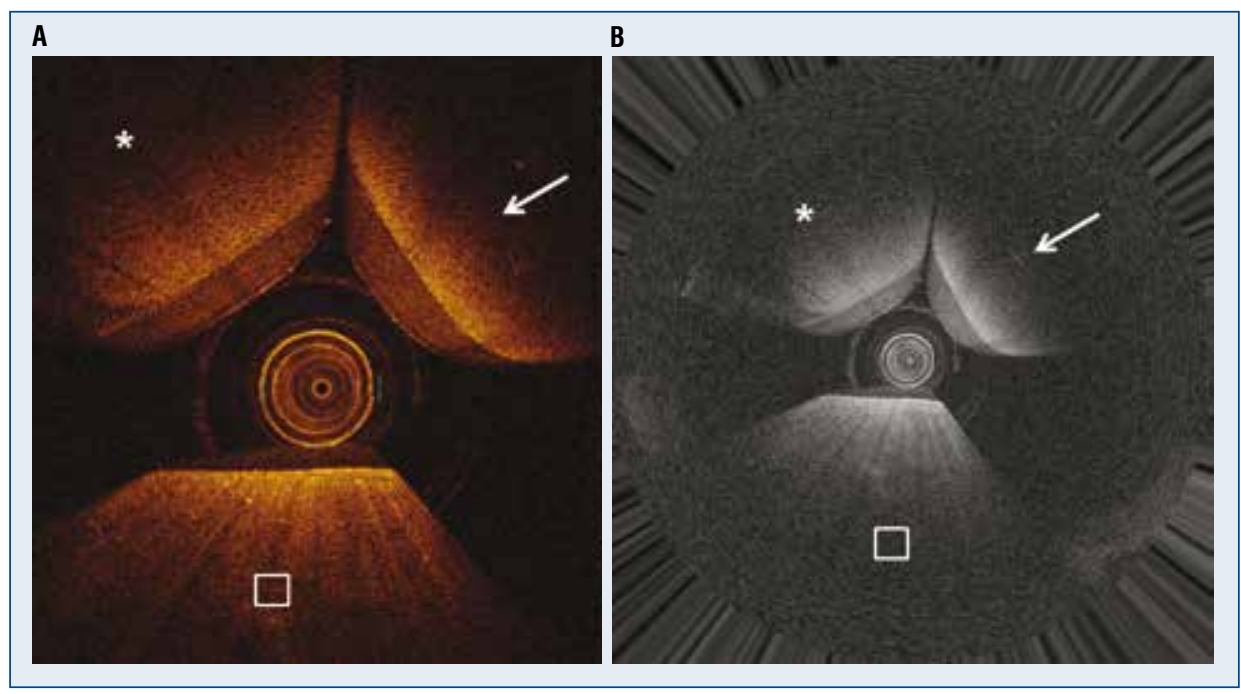

Figure 1. Optical coherence tomography (OCT) imaging of the test samples. Example of an OCT cross-section selected for the intensity analysis, as seen in the OCT console (A) and corresponding to the log-transformed image of the raw OCT signal in Cartesian view, as seen in the interface of the quantification software (B). The cross-section shows a pair of reaction tubes containing cell pellets of RAW 264.7 non-labelled macrophages $\left({ }^{*}\right)$ and very small superparamagnetic oxydised nanoparticles-loaded macrophages (arrow), displayed over a grid ( $\square$ ).

fication step was repeated 3 times and the particles were finally re-dispersed in PBS buffer (pH: 7.4).

\section{Sample preparation}

Cells from the macrophage cell line RAW 264.7, derived from mice peritoneal macrophages transformed by the AMLV (Abelson Murine Leukemia Virus), were cultured in Dulbecco's Modified Eagle's Medium (DMEM), complemented with $10 \%$ of fetal bovine serum (FBS, Gibco), $1 \%$ penicillin-streptomycin (penicillin 10,000 units/mL, streptomycin $10 \mathrm{mg} / \mathrm{mL}$; Sigma-Aldrich) and incubated at $37^{\circ} \mathrm{C}$ in a $5 \%$ carbon dioxide humidified atmosphere. Cells were regularly passaged until reaching more than $80 \%$ confluence and medium was exchanged every 2 days.

Nanoparticles uptake was performed with macrophages seeded at $100,000 \mathrm{cells} / \mathrm{cm}^{2}$ in $75 \mathrm{~cm}^{2}$ tissue culture flasks to allow overnight adherent cell recovery and proliferation. Following day, growth medium was removed and culture flasks were washed twice with phosphate buffered saline (PBS) followed by addition of NP solution in DMEM without phenol red and complemented with $1 \%$ FBS. Cells were incubated with VSOP ( $1 \mathrm{mM}$ Fe final concentration) for $24 \mathrm{~h}$ at $37^{\circ} \mathrm{C}$ in a $5 \%$ carbon dioxide humidified atmosphere. Nonbound nanoparticles were removed by washing 3 times with PBS, centrifugation (1,000 rpm for $5 \mathrm{~min}$ ) and cell passage into a $25 \mathrm{~cm}^{2}$ at 100,000 cells $/ \mathrm{cm}^{2}$. Overnight cell recovery was allowed un- der cell growth conditions before cell pellet collection. Five different test samples were precipitated in $0.2 \mathrm{~mL}$ transparent polypropylene thin-walled PCR tubes (Applied Biosystems) to study their optical properties. These samples consisted of 2 cell pellets for RAW 264.7, containing $4 \times 10^{6}$ cells in a volume of $10 \mu \mathrm{L}: 1$ ) Cell pellet with non-labelled macrophages, used as control; 2) Macrophages incubated with VSOP $1 \mathrm{mM}$ Fe. In order to study the effect of cellular accumulation of the nanoparticles on the optical backscattering and attenuation, an additional control sample containing VSOP in suspension without cells was arranged: 3) VSOP $1 \mathrm{mM} \mathrm{Fe}$ in suspension $(300 \mu \mathrm{L})$.

\section{OCT acquisition}

A Dragonfly optical catheter (Saint Jude Medical, St. Paul, Minnesota, USA) was fixed on the surface of a grid and the PCR tubes containing the test samples were set pairwise at both sides of the optical catheter, aligning the walls of their tapered tip in parallel with the catheter, so the test samples could be imaged through the part of the tube where the wall was thinnest. A pullback was then acquired at open air with an Ilumien-Optis Fourier-domain OCT system (Saint Jude Medical, St. Paul, Minnesota, USA) at a rotation speed of $180 \mathrm{~Hz}$ and a pullback speed of $20 \mathrm{~mm} / \mathrm{s}$ (Fig. 1).

The following pairs were selected for the OCT acquisition, in order to enable a direct comparison of their optical properties: 1) Non-labelled mac- 
rophages vs. macrophages labelled with VSOP, 2) Macrophages labelled with VSOP vs. VSOP in suspension.

\section{Light intensity analysis}

A representative cross-section was selected for each pair of tubes, in which both test samples were visible at the highest quality. Using the polar view of the log-transformed OCT signal, quantification frames of exactly $400 \times 50$ pixels were placed in each test sample at the closest point to the optical catheter, starting at and including the interface with the PCR tube and extending into the depth of the study sample up to the complete attenuation of the optical signal (Fig. 2), thus ensuring a perfect alignment of the measurements with the irradiation of the NIR beam. The coordinates of each quantification frame were registered.

The OCT images of the selected cross-sections were then displayed as raw linear signal in the polar view, and quantification frames of $400 \times 50$ pixels were then placed at exactly the same coordinates previously registered under guidance of the logtransformed signal. The average light intensity values of the 50 transversal pixels were then longitudinally recorded along the 400 pixels. The absolute intensity values were then normalised using the intensity of the backscattering at the corresponding PCR tube: $50 \times 20 \mathrm{~mm}$ control frames were placed immediately proximal to the each sample quantification frame, with neither solution of continuity nor overlap (Fig. 2); the absolute light intensity values were then divided by the average intensity measured in the $50 \times 20$ control frame at the PCR tube. This way, the normalised intensity values express the light intensity as the n-fold value with respect to the intensity of the corresponding PCR tube: a normalised intensity of 1 is equivalent to the same light intensity as the PCR tube, normalised values between 0 and 1 correspond to intensity values lower than the intensity of the tube, whilst values $>1$ correspond to intensity values above the intensity of the tube. The backscattering is estimated as the peak intensity value of the sample, whereas the attenuation is estimated as the number of pixels between the peak intensity value and the normalised intensity of 1 (peak-to-one).

\section{Results}

\section{Light intensity analysis with OCT}

Very small superparamagnetic iron oxide particles-loaded macrophages show enhanced backscattering in conventional log-transformed
OCT imaging, i.e. they appear brighter than unlabelled macrophages, so as to be noticeable by the unaided eye (Fig. 3). This qualitative observation is substantiated by light intensity analysis of the linear raw OCT signal: VSOP-loaded macrophages have higher backscattering than the corresponding unlabelled macrophages (peak normalised intensity 6.30 vs. 3.15 ) with also slightly higher attenuation of the signal (peak-to-one 61 vs. 66 pixels) (Table 1, Fig. 4). VSOP in suspension do not have any significant backscattering (it is lower than the PCR tube containing the test sample: peak normalised intensity 0.94) (Table 1, Fig. 4) in the light intensity analysis.

\section{Discussion}

The main findings of this study can be summarised as follows: 1) VSOP-loaded macrophages show more intense optical backscattering than ordinary unlabelled macrophages in the NIR wavelengths used in OCT. 2) Imaging of macrophages in OCT can be enhanced by superparamagnetic iron oxide nanoparticles, resulting in twice as much backscattering as the unlabelled macrophages, but only a slight increase in the signal attenuation.

These results confirm the driving hypothesis of this study and encourage the further development of nanoparticles-based methods for visualisation of macrophages and inflammatory cells in the coronary vessels by means of OCT. Since the backscattering of a structure determines how bright we will see that structure in OCT, whilst its attenuation determines whether we will see shadowing behind that structure or not, a technique which doubles the backscattering with only a minimal increase in the attenuation is expected to render a more-than-twice brighter image (depending on the log-transformation) with somewhat more shadowing. Figure 3 presents a paradigmatic example.

Interestingly this backscattering enhancement depends strictly on the cellular accumulation of the VSOP within the macrophages. A similar concentration of VSOP in suspension, without cells, results in no significant backscattering at all in the light intensity analysis with OCT (Table 1, Fig. 1). The uptake and accumulation of VSOP in phagolysosomal structures of the cytosol of the macrophage might increase the local concentration of the nanoparticles as to form large VSOP aggregates that are effective scatterers [21], since the scattering increases with the $6^{\text {th }}$ power of the particle size according to Rayleigh's theory [24]. The fact that this macrophagic enhancement comes 


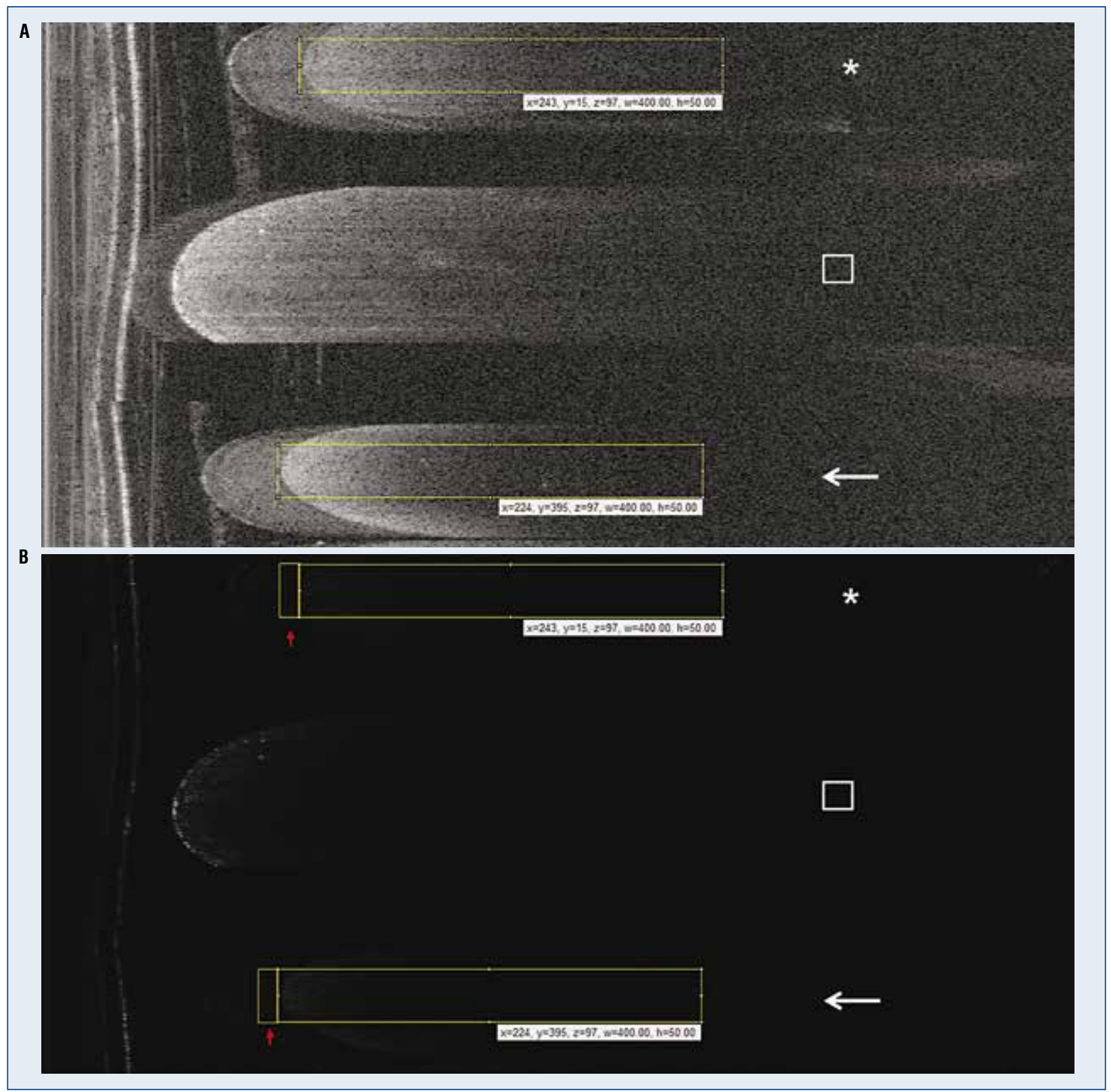

Figure 2. Light intensity analysis of the selected optical coherence tomography cross-sections. Polar view of the cross-section showed in Figure 2. The log-transformed image $(A)$ is used to place the quantification frames $(400 \times 50)$ for the sample of non-labelled macrophages $\left({ }^{*}\right)$ and for the sample of very small superparamagnetic iron oxide particles-loaded macrophages (arrow) at the point in which each test tube is closest to the optical catheter. The frames are aligned with the optical beam and start at the interface tube-sample up to the depth of the sample, where the optical signal is completely attenuated. The coordinates of the quantification frames were registered and replicated in the linear raw signal (B) for the intensity analysis. The intensity of a small $20 \times 50$ area at the proximal tip of each quantification frame (red arrows), corresponding to the backscattering of the PCR tube, was measured and used to normalise the absolute intensity values.

only at the cost of a minimal increase in the attenuation of the signal is also important for OCT imaging, because an excess of shadowing could preclude the appropriate imaging of other components of the plaque, thus limiting the usefulness of this technique. Nonetheless we must keep in mind that the accumulation of unlabelled macrophages attenuates itself the signal remarkably, so the true impact of this additional increase in attenuation needs to be defined after in vivo studies. 


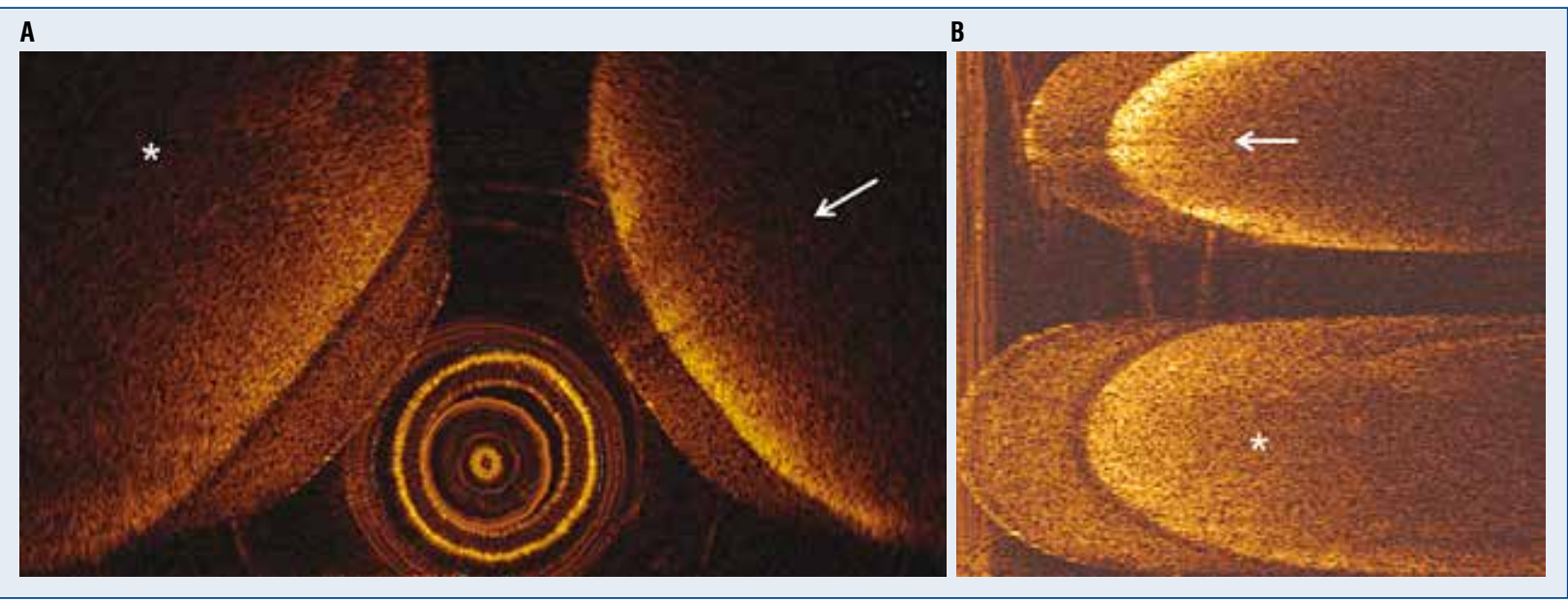

Figure 3. Optical coherence tomography (OCT) imaging of very small superparamagnetic iron oxide particles (VSOP)-loaded and unlabelled macrophages. Cell pellets containing unlabelled RAW 264.7 macrophage cells $\left({ }^{*}\right)$ and macrophage cells loaded with VSOP (arrow), as seen in conventional OCT: Cartesian view (A) and polar view (B). VSOP-loaded macrophages show enhanced backscattering, i.e. they appear brighter than the control unlabelled macrophages, so as to be noticeable with the unaided eye.

Table 1. Light intensity analysis of the linear raw optical coherence tomography signal. Backscattering is estimated as the peak normalised intensity value of the sample. Attenuation is estimated as the number of pixels between the peak intensity and the first normalised intensity value below 1.

\begin{tabular}{clcc}
\hline & & $\begin{array}{c}\text { Backscattering } \\
\text { (peak intensity) }\end{array}$ & $\begin{array}{c}\text { Attenuation } \\
\text { (peak-to-1) }\end{array}$ \\
\hline Pair 1 & Macrophages (non-labelled) & 3.15 & 66 \\
& VSOP-labelled macrophages & 6.30 & 61 \\
Pair 2 & Macrophages (non-labelled) & 3.22 & 73 \\
& VSOP suspension (no cells) & 0.94 & NA \\
\hline
\end{tabular}

VSOP - macrophages loaded with very small superparamagnetic iron oxide particles; VSOP suspension — very small superparamagnetic iron oxide particles in suspension, without cells

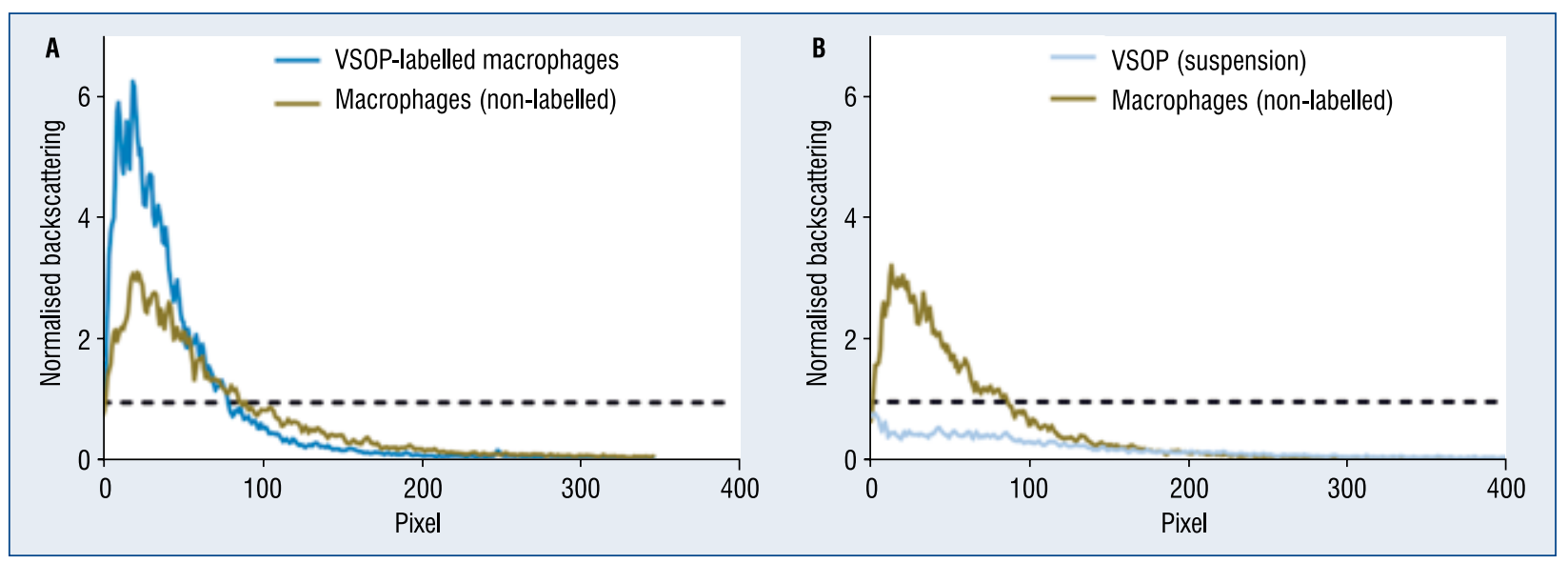

Figure 4. A, B. Light intensity analysis of the linear raw optical coherence tomography (OCT) signal. The light intensity was measured in quantification frames of $400 \times 50$ pixels. The $y$-axis shows the average intensity in the 9 transversal pixels, displayed along the 400 longitudinal pixels (x-axis), from the interface with the PCR tube (pixel 1) to the furthest part of the test sample from the optical catheter (pixel 400); VSOP — very small superparamagnetic iron oxide particles. 
A technique for macrophagic enhancement in conventional OCT might open new perspectives for in vivo imaging of the atherosclerotic plaque and contribute to understanding the role of inflammation in plaque progress and stability. The possibility of sequential assessment of these dynamic processes is particularly appealing and likely to offer new insights on an old disease. It is important to highlight that accumulation of macrophages can be already seen in conventional intracoronary OCT without need of any enhancer [17], even represented in 3-dimensional rendering of the vessel [27], however the degree of consensus among different investigators regarding the reliability of OCT for the detection of macrophages is still low [18]. The use of superparamagnetic iron oxide nanoparticles for macrophagic enhancement might improve the sensitivity and specificity of OCT for the detection of these cells and eventually contribute to improve the level of consensus among the experts. Moreover, the detection of macrophages can be certainly of interest for other coronary processes with a strong inflammatory component, like spontaneous coronary artery dissection [28], cardiac allograft vasculopathy [29] or in-stent restenosis [30].

The implementation of nanoparticles as macrophagic enhancers would be feasible and simple: it only requires a previous intravenous injection of the nanoparticle. VSOP might have interesting pharmacokinetic characteristics as compared with other nanoparticles. VSOP are directly taken up by endothelial cells and macrophages, cumulate in phagolysosomes and reach sufficient levels for imaging in less than $3 \mathrm{~h}$ after administration $[20,21]$. The attractive pharmacokinetic profile of VSOP must be however balanced with the lack of approval for human use yet, although they are currently being tested for imaging in magnetic resonance $[22,23]$.

\section{Limitations of the study}

The possibility of an irregular distribution of the cells or the particles in the pellets cannot be completely ruled out. This phenomenon could partially explain some of the differences in backscattering and attenuation observed between the same elements imaged in different pairs. However the results are consistent enough as to support the driving hypothesis and the differences between pairs are most likely due to the many possible factors determining the absolute backscattering of a structure besides its intrinsic optical properties (distance to the catheter, beam incidence, etc.).
Indeed the need to control all these factors led to the design of the study as paired comparisons of the samples that were simultaneously imaged.

This is a cellular ex vivo study proving the usefulness of nanoparticles for macrophagic enhancement with conventional OCT. The real potential and limitations of this method must be defined in detail by means of in vivo animal and ultimately clinical studies. The possibility of nonselective imaging can limit the applications of this new technique, particularly in the case of VSOP, which also cumulate in endothelial cells [31] and might interfere with the overall quality of the OCT acquisition at some extent.

\section{Conclusions}

Very small iron oxide particles increase significantly the optical backscattering of macrophages in the NIR regions of the spectrum, with only a minimal increase in the signal attenuation. This finding enables the enhancement of macrophages in conventional OCT imaging with a feasible and easily implementable methodology.

\section{Conflict of interest: None declared}

\section{References}

1. Murray CJ, Lopez AD. Mortality by cause for eight regions of the world: Global Burden of Disease Study. Lancet. 1997; 349(9061): 1269-1276, doi: 10.1016/S0140-6736(96)07493-4, indexed in Pubmed: 9142060.

2. Murray CJ, Lopez AD. Alternative projections of mortality and disability by cause 1990-2020: Global Burden of Disease Study. Lancet. 1997; 349(9064): 1498-1504, doi: 10.1016/S01406736(96)07492-2, indexed in Pubmed: 9167458.

3. Mathers CD, Boerma T, Ma Fat D. Global and regional causes of death. Br Med Bull. 2009; 92: 7-32, doi: 10.1093/bmb/ldp028, indexed in Pubmed: 19776034.

4. Falk E, Shah PK, Fuster V. Coronary Plaque Disruption. Circulation. 1995; 92(3): 657-671, doi: 10.1161/01.cir.92.3.657.

5. Burke AP, Farb A, Malcom GT, et al. Coronary risk factors and plaque morphology in men with coronary disease who died suddenly. N Engl J Med. 1997; 336(18): 1276-1282, doi: 10.1056/ NEJM199705013361802, indexed in Pubmed: 9113930.

6. Lendon CL, Davies MJ, Born GV, et al. Atherosclerotic plaque caps are locally weakened when macrophages density is increased. Atherosclerosis. 1991; 87(1): 87-90, indexed in Pubmed: 1872926.

7. Moreno PR, Falk E, Palacios IF, et al. Macrophage infiltration in acute coronary syndromes. Implications for plaque rupture. Circulation. 1994; 90(2): 775-778, indexed in Pubmed: 8044947.

8. Libby P, Geng YJ, Aikawa M, et al. Macrophages and atherosclerotic plaque stability. Curr Opin Lipidol. 1996; 7(5): 330-335, indexed in Pubmed: 8937525.

9. Dirksen MT, van der Wal AC, van den Berg FM, et al. Distribution of inflammatory cells in atherosclerotic plaques relates 
to the direction of flow. Circulation. 1998; 98(19): 2000-2003, indexed in Pubmed: 9808596.

10. Moreno PR, Bernardi VH, Lopez-Cuellar J, et al. Macrophages, smooth muscle cells, and tissue factor in unstable angina: implications for cell-mediated thrombogenicity in acute coronary syndromes. Circulation. 1996; 94(12): 3090-3097, doi: 10.1161/01.cir.94.12.3090.

11. Libby P, Tabas I, Fredman G, et al. Inflammation and its resolution as determinants of acute coronary syndromes. Circ Res. 2014; 114(12): 1867-1879, doi: 10.1161/CIRCRESAHA.114.302699, indexed in Pubmed: 24902971.

12. Davies MJ, Richardson PD, Woolf N, et al. Risk of thrombosis in human atherosclerotic plaques: role of extracellular lipid, macrophage, and smooth muscle cell content. Br Heart J. 1993; 69(5): 377-381, indexed in Pubmed: 8518056.

13. van der Wal AC, Becker AE, van der Loos CM, et al. Site of intimal rupture or erosion of thrombosed coronary atherosclerotic plaques is characterized by an inflammatory process irrespective of the dominant plaque morphology. Circulation. 1994; 89(1): 36-44, doi: 10.1161/01.cir.89.1.36.

14. Galis ZS, Sukhova GK, Kranzhöfer R, et al. Macrophage foam cells from experimental atheroma constitutively produce matrixdegrading proteinases. Proc Natl Acad Sci U S A. 1995; 92(2): 402-406, indexed in Pubmed: 7831299.

15. Lee RT, Schoen FJ, Loree HM, et al. Circumferential stress and matrix metalloproteinase 1 in human coronary atherosclerosis. Implications for plaque rupture. Arterioscler Thromb Vasc Biol. 1996; 16(8): 1070-1073, indexed in Pubmed: 8696948.

16. Gutiérrez-Chico JL, Alegría-Barrero E, Teijeiro-Mestre R, et al. Optical coherence tomography: from research to practice. Eur Heart J Cardiovasc Imaging. 2012; 13(5): 370-384, doi: 10.1093/ ehjci/jes025, indexed in Pubmed: 22330231.

17. Tearney GJ, Yabushita H, Houser SL, et al. Quantification of macrophage content in atherosclerotic plaques by optical coherence tomography. Circulation. 2003; 107(1): 113-119, indexed in Pubmed: 12515752.

18. Tearney GJ, Regar E, Akasaka T, et al. Consensus standards for acquisition, measurement, and reporting of intravascular optical coherence tomography studies: a report from the International Working Group for Intravascular Optical Coherence Tomography Standardization and Validation. J Am Coll Cardiol. 2012; 59(12): 1058-1072, doi: 10.1016/j.jacc.2011.09.079, indexed in Pubmed: 22421299.

19. Herborn CU, Vogt FM, Lauenstein TC, et al. Magnetic resonance imaging of experimental atherosclerotic plaque: comparison of two ultrasmall superparamagnetic particles of iron oxide. J Magn Reson Imaging. 2006; 24(2): 388-393, doi: 10.1002/ jmri.20649, indexed in Pubmed: 16791857.

20. Wagner S, Schnorr J, Ludwig A, et al. Contrast-enhanced MR imaging of atherosclerosis using citrate-coated superparamagnetic iron oxide nanoparticles: calcifying microvesicles as imaging target for plaque characterization. Int J Nanomedicine. 2013; 8: 767-779, doi: 10.2147/JJN.S38702, indexed in Pubmed: 23450179 .
21. Scharlach C, Kratz H, Wiekhorst F, et al. Synthesis of acidstabilized iron oxide nanoparticles and comparison for targeting atherosclerotic plaques: evaluation by MRI, quantitative MPS, and TEM alternative to ambiguous Prussian blue iron staining. Nanomedicine. 2015; 11(5): 1085-1095, doi: 10.1016/j. nano.2015.01.002, indexed in Pubmed: 25659644.

22. Wagner M, Wagner S, Schnorr J, et al. Coronary MR angiography using citrate-coated very small superparamagnetic iron oxide particles as blood-pool contrast agent: initial experience in humans. J Magn Reson Imaging. 2011; 34(4): 816-823, doi: 10.1002/ jmri.22683, indexed in Pubmed: 21769977.

23. Taupitz M, Wagner S, Schnorr J, et al. Phase i clinical evaluation of citrate-coated monocrystalline very small superparamagnetic iron oxide particles as a new contrast medium for magnetic resonance imaging. Invest Radiol. 2004; 39(7): 394-405, doi: 10.1097/01.rli.0000129472.45832.b0.

24. Gregory J. Monitoring particle aggregation processes. Adv Colloid Interface Sci. 2009; 147-148: 109-123, doi: 10.1016/j. cis.2008.09.003, indexed in Pubmed: 18930173.

25. Scharlach C, Warmuth C, Schellenberger E. Determination of blood circulation times of superparamagnetic iron oxide nanoparticles by $\mathrm{T}^{*} *$ relaxometry using ultrashort echo time (UTE) MRI. Magn Reson Imaging. 2015; 33(9): 1173-1177, doi: 10.1016/j.mri.2015.06.017, indexed in Pubmed: 26119420.

26. Khalafalla S, Reimers G. Preparation of dilution-stable aqueous magnetic fluids. IEEE Transactions on Magnetics. 1980; 16(2): 178-183, doi: 10.1109/tmag.1980.1060578.

27. Tearney GJ, Waxman S, Shishkov M, et al. Three-dimensional coronary artery microscopy by intracoronary optical frequency domain imaging. JACC Cardiovasc Imaging. 2008; 1(6): 752 -761, doi: 10.1016/j.jcmg.2008.06.007, indexed in Pubmed: 19356512.

28. Saw J, Ricci D, Starovoytov A, et al. Spontaneous coronary artery dissection: prevalence of predisposing conditions including fibromuscular dysplasia in a tertiary center cohort. JACC Cardiovasc Interv. 2013; 6(1): 44-52, doi: 10.1016/j.jcin.2012.08.017, indexed in Pubmed: 23266235.

29. Mehra MR, Crespo-Leiro MG, Dipchand A, et al. International Society for Heart and Lung Transplantation working formulation of a standardized nomenclature for cardiac allograft vasculopathy-2010. J Heart Lung Transplant. 2010; 29(7): 717-727, doi: 10.1016/j.healun.2010.05.017, indexed in Pubmed: 20620917.

30. Moreno PR, Bernardi VH, López-Cuéllar J, et al. Macrophage infiltration predicts restenosis after coronary intervention in patients with unstable angina. Circulation. 1996; 94(12): 3098-3102, indexed in Pubmed: 8989115.

31. Scharlach C, Müller L, Wagner S, et al. LA-ICP-MS Allows Quantitative Microscopy of Europium-Doped Iron Oxide Nanoparticles and is a Possible Alternative to Ambiguous Prussian Blue Iron Staining. J Biomed Nanotechnol. 2016; 12(5): 1001-1010, indexed in Pubmed: 27305821. 